\title{
Increased copy number of mitochondrial DNA predicts poor prognosis of esophageal squamous cell carcinoma
}

\author{
HENG LI ${ }^{1,2}$, ZHUFANG TIAN ${ }^{2}$, YONG ZHANG ${ }^{3}$, QI YANG ${ }^{1}$, BINGYIN SHI ${ }^{1}$, PENG HOU ${ }^{1}$ and MEIJU JI ${ }^{4}$ \\ ${ }^{1}$ Department of Endocrinology, The First Affiliated Hospital of Xi'an Jiaotong University, Xi'an, Shaanxi 710061; \\ ${ }^{2}$ Department of Endocrinology, Xi'an Central Hospital, Xi'an, Shaanxi 710003; ${ }^{3}$ Department of Thoracic Surgery; \\ ${ }^{4}$ Center for Translational Medicine, The First Affiliated Hospital of Xi'an Jiaotong University, Xi'an, Shaanxi 710061, P.R. China
}

Received November 18, 2016; Accepted September 22, 2017

DOI: $10.3892 / \mathrm{ol} .2017 .7416$

\begin{abstract}
Change in mitochondrial DNA (mtDNA) copy number has been reported in esophageal squamous cell carcinoma (ESCC). However, its prognostic implication in ESCC remains largely unknown. Using reverse transcription-quantitative PCR assay, the mtDNA copy number was assessed in a cohort of patients with ESCC (n=141) and normal esophageal tissues $(n=45)$, and the association between variable mtDNA levels and clinical outcomes of patients with ESCC were studied. Data revealed that ESCC patients exhibited an increased mtDNA content compared to control subjects. Furthermore, increased mtDNA content was associated with a significantly increased risk of cancer-associated mortality. This molecular event was associated with poorer survival in patients with ESCC, and was an independent predictor of patient survival. Data demonstrated that increased mtDNA content is a common genetic event in ESCC and may be a predictive factor of poor prognosis for ESCC patients.
\end{abstract}

\section{Introduction}

Esophageal cancer is a notable global health problem, although its incidence varies throughout the world (1-6). According to histological classification, esophageal cancer is categorized into esophageal squamous cell carcinoma (ESCC) and esophageal adenocarcinoma (EAC), each of which possesses different risk factors and pathogenesis (6). ESCC frequently occurs in China and other Asian countries, as well as in certain parts of Iran and South Africa, whereas the incidence of EAC is markedly increased in Western countries, including the USA and a number of European

Correspondence to: Professor Meiju Ji, Center for Translational Medicine, The First Affiliated Hospital of Xi'an Jiaotong University, 277 Yanta West Road, Xi'an, Shaanxi 710061, P.R. China

E-mail:mjji0409@163.com

Key words: mitochondrial DNA, copy number, esophageal squamous cell carcinoma, reverse transcription-quantitative polymerase chain reaction, clinical outcomes countries (6). EAC accounts for $\sim 66 \%$ of esophageal cancer cases in the USA, whereas ESCC currently accounts for $90 \%$ of worldwide esophageal cancer cases (6). Thus far, the majority of esophageal cancer cases are diagnosed at advanced stages of the disease, which makes curative surgery unfeasible, leading to a poor overall prognosis in patients with esophageal cancer. Thus, novel strategies and biomarkers are necessary in order to provide early detection, prevention or prediction of treatment responses and the prognosis of esophageal cancer.

In all nucleated cells mitochondria are powerhouses, converting oxygen and glucose into adenosine triphosphate (ATP) through oxidative phosphorylation (7). The mitochondrion possesses its own mitochondrial DNA (mtDNA), which is ring-like and double-stranded. Human mtDNA contains non-coding and coding regions, and comprises $\sim 16,569$ base pairs (7). The 13 polypeptides encoded by the coding region constitute respiratory enzyme complexes, two ribosomal RNAs and a set of 22 transfer RNAs, all of which are necessary for the synthesis of certain mitochondrial peptides (8). The displacement loop (D-loop) is a non-coding region serving a function in mtDNA replication and transcription. Thus, D-loop mutations may lead to alterations in mtDNA content or gene expression $(9,10)$. Cells typically contain high levels of mtDNA, and the majority of the copies are determinated at birth (11).

Compared with nuclear DNA, the mitochondrial genome is more susceptible to mutation and is sensitive to oxidative damage $(8,12)$. Thus, mitochondrial dysfunction is hypothesized to have a marked influence on tumorigenesis and progression. The copy number of mtDNA is increased during progression of ESCC, glioma, lung and laryngeal cancer, whereas the copy number of mtDNA is decreased in bladder, breast and gastric cancer, and hepatocellular carcinomas, compared with non-cancerous tissues (8,12-16). Furthermore, accumulated evidence has demonstrated that in peripheral blood lymphocytes, altered mtDNA content is closely associated with the risk of certain types of cancer (17-24). However, the association between various copy numbers of mtDNA and prognosis in ESCC remains unresolved. In the present study, altered mtDNA content in normal esophageal tissues and ESCC tissues was investigated using reverse transcription-quantitative polymerase chain reaction (RT-qPCR), and 
the associations between mtDNA content and clinicopathological features, and the prognosis of patients with ESCC were also explored.

\section{Materials and methods}

Patients and tissue samples. Approval for the present study was obtained from Human Ethics Committee and Institutional Review Board, Xi'an Jiaotong University (Xi'an, China). A total of 141 paraffin-embedded ESCC tissue samples were collected from the First Affiliated Hospital of Xi'an Jiaotong University (Xi'an, China) between January 2002 and September 2010. Additionally, 45 samples were obtained from esophagitis patients that underwent endoscopy for biopsy, and were used as control subjects. Patients with chemoradiotherapy were excluded. Written informed consent was obtained from each patient prior to surgery. The diagnosis of esophageal diseases was based on histological examination according to the World Health Organization diagnosis standard of esophageal diseases (25). The clinicopathological data of the patients was collected from their medical records and the results are presented in Table I.

DNA preparation. Serial sections were cut at $5 \mu \mathrm{m}$ from paraffin embedded tumor and control tissues. One section from each sample was stained by hematoxylin and eosin (H\&E) staining as described below. Briefly after deparaffinizing and hydrating the tissue sections, slides were incubated with hematoxylin solution in a staining jar for $10 \mathrm{~min}$ to stain the nuclei. Then the slides were transferred to a staining jar with running water until the water ran clear. Slides were then transferred to a staining jar with Eosin solution for $3 \mathrm{~min}$. Successively transfer the slides into staining jars with $70 \%$ ethanol for $20 \mathrm{sec}, 90 \%$ ethanol for $20 \mathrm{sec}, 100 \%$ ethanol for $1 \mathrm{~min}$ and xylene for $3 \mathrm{~min}$. Slides were mounted with xylene-based mounting media and covered with cover slides. The representative tumor tissue was examined by an expert surgical pathologist at The Department of Pathology of the First Affiliated Hospital of Xi'an Jiaotong University (Xi'an, China) for esophageal cancer. The tumor tissues were isolated through manual microdissection using an inverted microscope according to the H\&E marker. DNA was then isolated from the tumor tissues as described previously (26). Briefly, after a treatment for $12 \mathrm{~h}$ at room temperature with xylene to remove paraffin, the tissues were then subjected to digestion with $1 \%$ sodium dodecyl sulfate (SDS) and proteinase $\mathrm{K}$ at $48^{\circ} \mathrm{C}$ for $48 \mathrm{~h}$, with addition of several spiking aliquots of concentrated proteinase $\mathrm{K}$ to facilitate digestion. DNA was subsequently isolated using a standard phenol-chloroform extraction and ethanol precipitation protocol, and kept at $-80^{\circ} \mathrm{C}$ until use.

mtDNA content analysis. The relative mtDNA content of the patients with ESCC and the control subjects was measured using RT-qPCR as described previously (15). The specific primers and TaqMan probes for MT-NDI (mitochondrially encoded NADH dehydrogenase 1 ) and the $\beta$-actin gene were designed using Primer Express software (version 3.0; Applied Biosystems; Thermo Fisher Scientific, Inc., Waltham, MA, USA) (Table II). Each sample was prepared in triplicate. To standardize the input DNA, $\beta$-actin was measured in parallel as a reference gene.
Table I. Clinicopathological characteristics of patients with ESCC.

\begin{tabular}{lr}
\hline Characteristics & Value \\
\hline Gender, n (\%) & \\
Male & $111(78.7)$ \\
Female & $30(21.3)$ \\
Age, years & \\
Mean & 58.3 \\
SD & 8.5
\end{tabular}

Tumor localization, n (\%)

Upper esophagus

$32(22.7)$

Middle esophagus

Lower esophagus

Tumor size, n (\%)

$<3 \mathrm{~cm}^{3}$

$3-5 \mathrm{~cm}^{3}$

$>5 \mathrm{~cm}^{3}$

Differentiation, n (\%)

Well/moderate

$112(79.4)$

Poor/undifferentiated

29 (20.6)

Tumor invasion, n (\%)

T1

15 (10.6)

$\mathrm{T} 2$

26 (18.4)

$72(51.1)$

28 (19.9)

$\mathrm{T} 4$

$15(10.6)$

$73(51.8)$

$50(35.5)$

IV

No

N3 ( $\geq 16)$

Alive

(a)

mtDNA, mitochondrial DNA; ESCC, esophageal squamous cell carcinoma; SD, standard deviation; TNM, Tumor-Node-Metastasis; LNM, lymph, node and metastasis.

Normal leukocyte DNA was used in serial dilutions to establish standard curves. The relative mtDNA content of each sample was measured as described previously (18).

Statistical analysis. The copy number of mtDNA between patients with ESCC and control subjects was compared using 
Table II. Primer and TaqMan probe sequences used in this study.

\begin{tabular}{llll}
\hline Genes & Forward primer sequence $\left(5^{\prime}-3^{\prime}\right)$ & \multicolumn{1}{c}{ Probe sequence $\left(5^{\prime}-3^{\prime}\right)$} & Reverse primer sequence $\left(5^{\prime}-3^{\prime}\right)$ \\
\hline MT-ND1 & CCCCTAAAACCCGCCACATC & 6FAM-ACCCTCTACATCA & GTAGAAGAGCGATGGTGAGAGC \\
& & CGCCCCGACC-TAMRA & \\
$\beta$-actin & TCACCCACACTGTGCCCATC & 6FAM-ATGCCCTCCC & TCGGTGAGGATCTTCATGAGGTA \\
& TACGA & CCATGCCATCC-TAMRA & \\
\hline
\end{tabular}

MT-ND1, mitochondrially encoded NADH dehydrogenase 1; FAM, 6-carboxyfluorescein; TAMRA, tetramethylrhodamine.

a Mann-Whitney $U$ test. The association of mtDNA content with clinicopathological features was assessed using univariate analysis. Multivariate models were developed and adjusted according to age, tumor size, differentiation and lymph node metastasis. Survival time was determined from the day of primary tumor resection to the day of cancer-associated mortality or last clinical follow-up. The Kaplan-Meier estimator method was used to analyze patient survival stratified by mtDNA content variations and the difference between the Kaplan-Meier curves was assessed using a log-rank test. A multivariate Cox's proportional hazards regression model was applied to calculate the hazard ratio (HR) with $95 \%$ confidence intervals (CIs) for prognosis evaluation. A multivariate Cox's regression analysis was performed to assess the influence of mtDNA content on patient prognosis, which was independent of the number of lymph node metastases, tumor invasion and differentiation. Statistical analyses were performed using SPSS software (version 11.5; SPSS, Inc., Chicago, IL, USA). P<0.05 was considered to indicate a statistically significant difference.

\section{Results}

Relative mtDNA content in ESCC. RT-qPCR was performed to analyze the mtDNA copy number in 141 patients with ESCC and 45 control subjects. As presented in Fig. 1, the relative mean mtDNA content was significantly increased in patients with ESCC $(4.67 \pm 3.88$ copies $)$ compared with that in control subjects $(2.24 \pm 1.80$ copies $)(\mathrm{P}<0.001)$. The median values among patients with ESCC and control subjects were 3.15 copies (interquartile range between 0.53 and 18.28 copies) and 1.80 copies (interquartile range between 0.47 and 7.50 copies), respectively, suggesting that the majority of patients with ESCC had increased mtDNA content compared with control subjects, which was consistent with a previous study (5). The possible difference in mtDNA content in tumor tissues with regard to selected clinicopathological features was also evlaluated. As presented in Fig. 2, no significant differences were identified in mtDNA copy number with reagrd to sex, age, tumor localization, tumor size, differentiation, tumor invasion, Tumor-Node-Metastasis stage (27) and lymph node metastasis. The group of patients who sucumbed to the disease exhibited an increased mtDNA copy number compared with the group of patients who did not succumb. Although no statistically significant difference was observed, there was a notable trend toward a positive association between mtDNA copy number and survival status of patients with $\operatorname{ESCC}(\mathrm{P}=0.054)$.

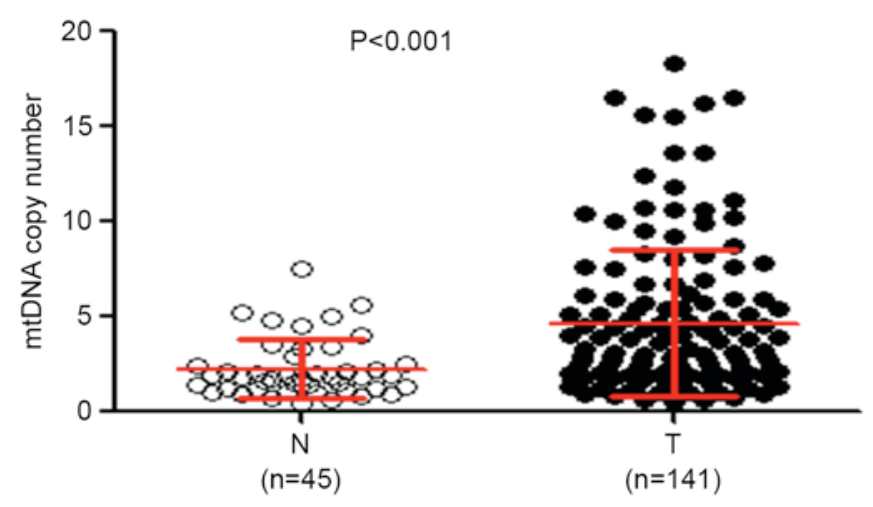

Figure 1. mtDNA copy number of each individual case of esophageal cancer and normal esophageal tissue. Reverse transcription-quantitative polymerase chain reaction was performed to analyze mtDNA copy number in patients with esophageal squamous cell carcinoma and normal esophageal tissues (control subjects). Horizontal lines represent mean \pm standard error of the mean. T, tumor tissues; N, control subjects; mtDNA, mitochondrial DNA.
Association between altered mtDNA content and clinicopathological features of ESCC. To investigate the association between mtDNA copy number and the clinicopathological features of ESCC, the median mtDNA copy number (3.14 copies) in the ESCC samples was selected as a threshold value. Using this threshold value, the ESCC samples were classified into two groups, namely increased mtDNA content ( $\geq 3.14$ copies) and decreased mtDNA content $(<3.14$ copies). As presented in Table III, mtDNA copy number variations was significantly associated with survival status in patients with ESCC. Compared with the control, the patients with ESCC exhibiting increased mtDNA content had an increased mortality rate [odds ratio (OR), 1.95; 95\% confidence interval (CI), 1.04-3.64; $\mathrm{P}=0.04]$. Multivariable logistic regression was performed to evaluate the independent association of variable mtDNA copy number with age, tumor size, differentiation and lymph node metastasis. As presented in Table IV the patients with increased mtDNA copy number had a 2.41 times higher risk of mortality than those with decreased mtDNA copy number. The data identified that increased mtDNA copy number remained positively associated with survival status following adjustment (OR, 2.41; 95\% CI, 1.13-5.13; $\mathrm{P}=0.02)$.

Effect of increased mtDNA content on prognosis of ESCC. The Kaplan-Meier estimator survival curves and log-rank test were used to assess the effect of altered mtDNA content 

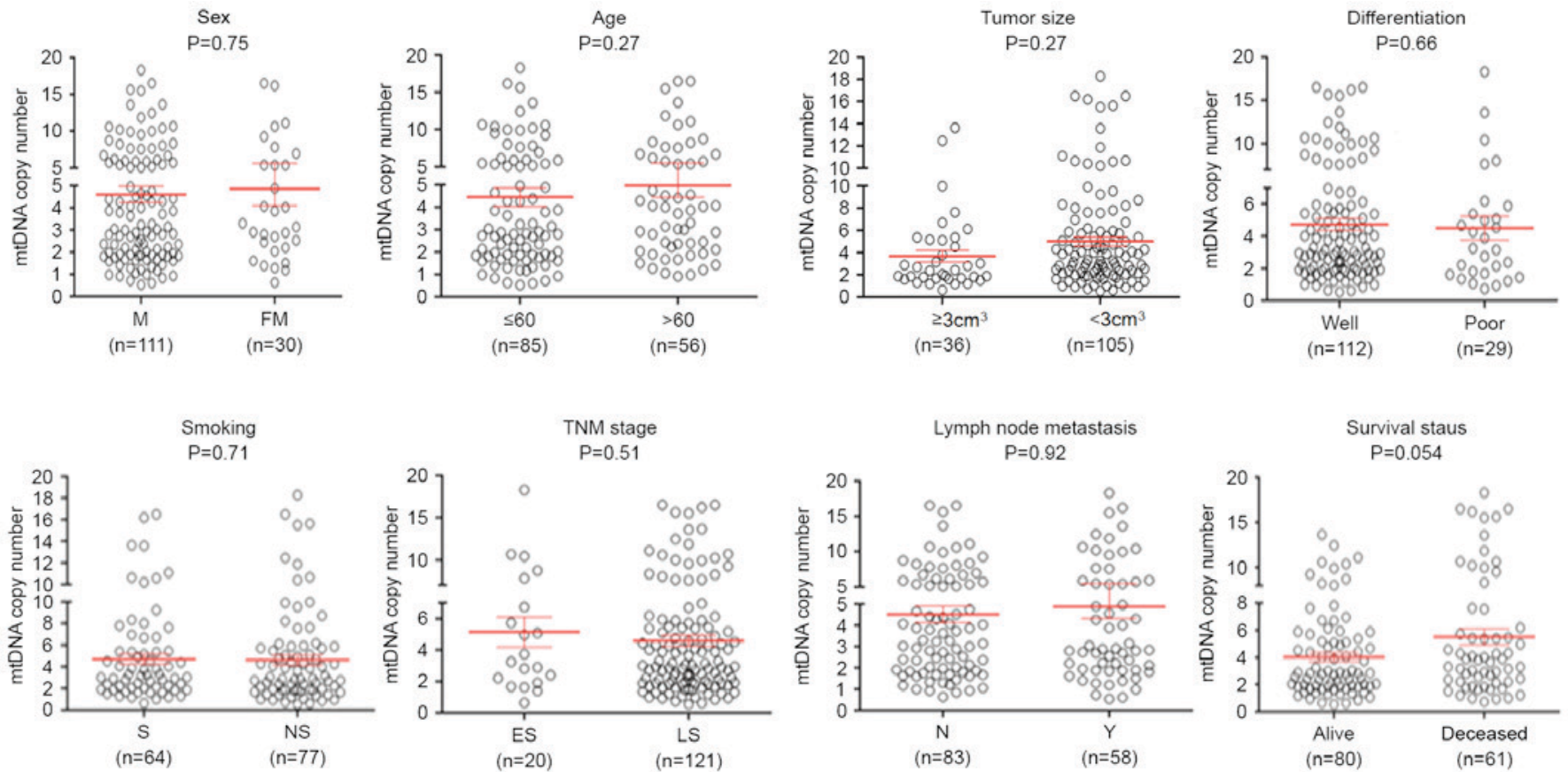

Figure 2. Association of mtDNA copy number with clinicopathological characteristics in ESCC. The copy number of mtDNA was analyzed using reverse transcription-quantitative polymerase chain reaction. Circles represent the mtDNA copy number of each case. Horizontal lines represent mean \pm standard error of the mean. Sample means were compared using the Mann-Whitney U test. mtDNA, mitochondrial DNA; ESCC, esophageal squamous cell carcinoma; FM, female; M, male; well, well/moderate differentiation; poor, poor/undifferentiated; ES, early-stage; LS, late-stage; S, smoking; NS, non-smoking; N, no metastasis present; Y, yes metastasis present; TNM, Tumor-Node-Metastasis.

Table III. Copy number variations of mitochondrial DNA in esophageal squamous cell carcinoma: Univariate associations with clinicopathological characteristics.

\begin{tabular}{lcc}
\hline Characteristics & Odds ratio (95\% CI) & P-value \\
\hline Sex & $0.98(0.44-2.20)$ & 0.97 \\
Age, years $^{\mathrm{a}}$ & $1.37(0.71-2.66)$ & 0.35 \\
Tumor localization $^{\mathrm{b}}$ & $0.90(0.57-1.41)$ & 0.64 \\
${\text { Tumor size, } \mathrm{cm}^{3 \mathrm{c}}}_{\text {Differentiation }^{\mathrm{d}}}$ & $1.87(0.83-4.20)$ & 0.13 \\
Tumor invasion $^{\mathrm{e}}$ & $1.33(0.76-2.33)$ & 0.33 \\
TNM stage $^{\mathrm{f}}$ & $1.31(0.48-3.55)$ & 0.59 \\
Lymph node metastasis $_{\text {Survival status }}^{\mathrm{g}}$ & $0.78(0.30-2.01)$ & 0.61 \\
& $0.74(0.38-1.46)$ & 0.39 \\
\hline
\end{tabular}

${ }^{\mathrm{a}} \mathrm{Age}$ (per 10 years); ${ }^{\mathrm{b}}$ Tumor localization (upper esophagus; middle esophagus; lower esophagus); ${ }^{\circ}$ Tumor size $\left(\leq 3 \mathrm{~cm}^{3} ;>3\right.$ and $\leq 5 \mathrm{~cm}^{3}$; $>5 \mathrm{~cm}^{3}$ ); ${ }^{\mathrm{d}}$ Differentiation (well or moderate; poor or undifferenti-

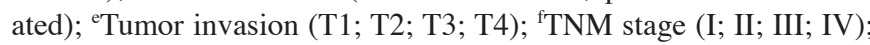
${ }^{\text {g}}$ Survival status (alive; deceased). CI, confidence interval; TNM, Tumor-Node-Metastasis.

on the survival time of patients with ESCC. As presented in Fig. 3, the patients with increased mtDNA copy number experienced shorter survival times than those with decreased mtDNA copy number (median survival time: 42.00 vs. 38.00 months; $\mathrm{P}=0.03$ ). Similarly, Cox's unvariate regression analysis demonstrated a marked association of increased
Table IV. Copy number variations of mitochondrial DNA in esophageal squamous cell carcinoma: Multivariable associations with clinicopathological characteristics.

\begin{tabular}{lcc}
\hline Characteristics & Odds Ratio (95\% CI) & P-value \\
\hline Sex & $1.07(0.43-2.64)$ & 0.89 \\
Age $^{\mathrm{a}}$ & $1.01(0.97-1.06)$ & 0.55 \\
Tumor size $^{\mathrm{b}}$ & $1.58(0.90-2.77)$ & 0.11 \\
Differentiation $^{\mathrm{c}}$ & $1.72(0.90-2.77)$ & 0.99 \\
Tumor invasion $^{\mathrm{d}}$ & $0.67(0.16-2.81)$ & 0.58 \\
TNM stage $^{\mathrm{e}}$ & $0.69(0.23-2.02)$ & 0.50 \\
Lymph node metastasis $^{\text {Survival status }}$ & $0.68(0.16-2.86)$ & 0.59 \\
Smoking $^{\mathrm{f}}$ & $2.41(1.13-5.13)$ & 0.02 \\
& $1.15(0.47-2.79)$ & 0.75 \\
\hline
\end{tabular}

${ }^{\mathrm{a}}$ Age (per 10 years); ${ }^{\mathrm{b}}$ Tumor size $\left(\leq 3 \mathrm{~cm}^{3} ;>3\right.$ and $\left.\leq 5 \mathrm{~cm}^{3} ;>5 \mathrm{~cm}^{3}\right)$; ${ }^{\mathrm{c}}$ Differentiation (well or moderate; poor or undifferentiated); ${ }^{\mathrm{d}}$ Tumor

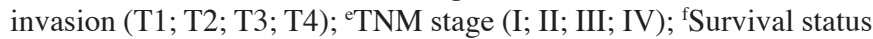
(alive; deceased); ${ }^{g}$ Smoking (smoking; no smoking). CI, confidence interval; TNM, Tumor-Node-Metastasis.

mtDNA copy number with poor patient survival [hazard ratio (HR), 1.76; 95\% CI, 1.06-2.97, $\mathrm{P}=0.03$; Table V] as well as age [HR, 1.75; 95\% CI, 1.05-2.91, $\mathrm{P}=0.03$ ] and TNM stage [HR, 2.40; 95\% CI, 1.44-3.99, P<0.01; Table V]. Additionally, Cox's multivariate regression analysis indicated that increased mtDNA content is a predictor of increased mortality for patients with ESCC (HR, 1.91; 95\% CI, 1.13-3.22; $\mathrm{P}=0.02$ ), 
Table V. Prognostic value of clinicopathological factors and copy number variation of mitochondrial DNA in univariate and multivariate Cox's regression analysis $(n=141)$.

\begin{tabular}{|c|c|c|c|c|}
\hline \multirow[b]{2}{*}{ Variables } & \multicolumn{2}{|c|}{ Univariate analysis } & \multicolumn{2}{|c|}{ Multivariate analysis } \\
\hline & Hazard ratio $(95 \% \mathrm{CI})$ & P-value & Hazard ratio $(95 \% \mathrm{CI})$ & P-value \\
\hline Copy number & & 0.03 & & 0.02 \\
\hline$<3.14$ & 1.00 & & 1.00 & \\
\hline$\geq 3.14$ & $1.76(1.06-2.97)$ & & $1.91(1.13-3.22)$ & \\
\hline Age, years ${ }^{\mathrm{a}}$ & & 0.03 & & 0.02 \\
\hline$<60$ & 1.00 & & 1.00 & \\
\hline$\geq 60$ & $1.75(1.05-2.91)$ & & $1.89(1.13-3.17)$ & \\
\hline Tumor invasion ${ }^{\mathrm{b}}$ & & 0.06 & & 0.18 \\
\hline $\mathrm{T} 1 / \mathrm{T} 2$ & 1.00 & & 1.00 & \\
\hline $\mathrm{T} 3 / \mathrm{T} 4$ & $2.65(0.96-7.30)$ & & $2.03(0.72-5.68)$ & \\
\hline TNM stage $\mathrm{c}^{\mathrm{c}}$ & & $<0.01$ & & $<0.01$ \\
\hline $\mathrm{I} / \mathrm{II}$ & 1.00 & & 1.00 & \\
\hline III/IV & $2.40(1.44-3.99)$ & & $2.54(1.50-4.30)$ & \\
\hline
\end{tabular}

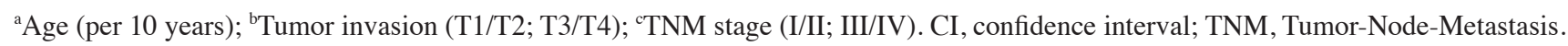

and is an independent variable with regard to the age and tumor stage (Table V).

\section{Discussion}

The primary function of the mitochondria is to produce the energy source ATP. Additionally, the mitochondria also serve functions in numerous cell processes, including cell differentiation, growth, death, cell cycle regulation and signal transduction (28). A previous study identified that extranuclear DNA, including mtDNA, and intranuclear genetic materials may have an effect on ESCC and the development of other tumors (8). It was identified that mtDNA content was significantly increased in patients with ESCC compared with control subjects, as supported by the results of a previous study (5). These data suggest that increased mtDNA content may be associated with esophageal tumorigenesis and the high mortality rate of patients with ESCC.

Changes in the mtDNA or mitochondrial dysfunction are frequently observed in tumor cells $(8,12-16)$. Several prior studies indicated that mtDNA content is increased in different types of cancer, including ESCC $(5,8,14-16)$. This proliferation of mtDNA is possibly a compensation mechanism induced by an energy shortage in tumor cells $(29,30)$. This is supported by a previous study, which demonstrated that relative mtDNA copy number increased progressively in normal esophageal mucosa, ESCC and metastatic lymph nodes, and that increased mtDNA content contributed to the increased bioenergetic capbility of the mitochondria, further promoting tumor invasion of ESCC (5).

In addition to its function supplying energy, increased mtDNA content may be associated with mtDNA escape in cancer. It has been identified that mtDNA escape is an essential exchange between the nuclear and mitochondrial

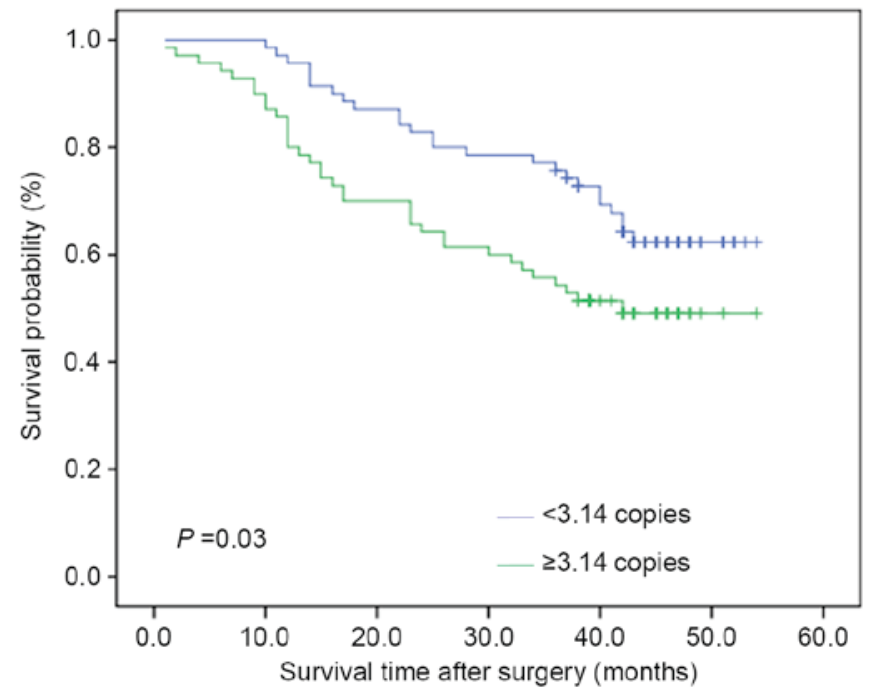

Figure 3. Kaplan-Meier estimator curves stratified by mtDNA copy number. Kaplan-Meier estimator survival analysis was performed according to copy number variations of mtDNA in this cohort of patients with ESCC. The data identified that increased mtDNA copy number ( $\geq 3.14$ copies) was markedly associated with decreased overall survival time of patients with ESCC. mtDNA, mitochondrial DNA; ESCC, esophageal squamous cell carcinoma.

genomes in yeast cells $(31,32)$. Similar to yeast, mammalian cells may require increased mtDNA content for the communication of genetic material between mitochondrial and nuclear genomes $(33,34)$. In line with this, a previous study utilizing fluorescence in situ hybridization demonstrated that mitochondrial sequence hybridization is observed in the nuclei more frequently in glioma tissues compared with normal brain tissues (33). Furthermore, it has been demonstrated that mtDNA sequences are able to interrupt intronic regions of certain 
cancer-associated genes, including the tumor suppressor gene mothers against decapentaplegic homolog 2 (35). mtDNA is able to cross with the nuclear genome in intronic and exonic regions $(36,37)$. Thus, increased mtDNA content may be symptomatic of an early genetic event contributing to tumorigenesis by supplying the energy for tumor cell growth and interrupting the function of specific tumor suppressor genes.

Since increased mtDNA content may contribute to tumorigenesis, a number of studies have been performed in order to explore the association between altered mtDNA content and clinical outcomes of patients with cancer $(38,39)$. In the present study, it was revealed that increased mtDNA content was associated with the survival status of patients with ESCC, and that the patients with high mtDNA content experienced significantly shorter survival times than those with low mtDNA content. When multivariate survival analysis was performed using Cox's regression model, it was once again identified that increased mtDNA content was associated with poorer patient survival rates. Taken together, these findings suggest that an altered mtDNA copy number may be a prognostic signature in ESCC.

A number of novel systems for copy number variation analysis have been developed, including magnetic nanoparticles-chemiluminescence detection (a combination of magnetic separation and chemiluminescence) (40), which has been used to analyze the copy numbers of porcine endogenous retroviruses and proviruses from Chinese Bama minipigs. This system possessess the advantages of being low-cost, simple, and providing high specificity and sensitivity compared with RT-qPCR, and as a result it, may be used in future studies (41).

In summary, the mtDNA content in patients with ESCC was investigated and it was demonstrated that increased mtDNA content may have an important impact on the tumorigenic phenotype of ESCC. The results of the present study also revealed that increased mtDNA copy number is an independent prognostic factor in ESCC, which may serve as a biomarker for predicting poor prognosis in patients with ESCC.

\section{Acknowledgements}

This study was supported by the National Natural Science Foundation of China (grant nos. 81402340 and 81472622), and the Fundamental Research Funds for the Central Universities (grant no. xjj2014147) and the Natural Science Basic Research Plan in Shaanxi Province of China (Program no. 2015JM8390).

\section{References}

1. Li JY, Liu BQ, Li GY, Chen ZL, Sun XI and Rong SD: Atlas of cancer mortality in the People's Republic of China. An aid for cancer control and research. Int J Epidemiol 10: 127-133, 1981.

2. Li JY: Cancer mapping as an epidemiologic research resource in China. Recent Results Cancer Res 114: 115-136, 1989.

3. Wu IC, Lu CY, Kuo FC, Tsai SM, Lee KW, Kuo WR, Cheng YJ, Kao EL, Yang MS and Ko YC: Interaction between cigarette, alcohol and betel nut use on esophageal cancer risk in Taiwan. Eur J Clin Invest 36: 236-241, 2006.

4. Lin CS, Chang SC, Wei YH, Chou TY, Wu YC, Lin HC, Wang LS and Hsu WH: Prognostic variables in thoracic esophageal squamous cell carcinoma. Ann Thorac Surg 87: 1056-1065, 2009.

5. Lin CS, Chang SC, Wang LS, Chou TY, Hsu WH, Wu YC and Wei YH: The role of mitochondrial DNA alterations in esophageal squamous cell carcinomas. J Thorac Cardiovascular Surg 139: 189-197.e4, 2010 .
6. Jemal A, Bray F, Center MM, Ferlay J, Ward E and Forman D: Global Cancer Statistics. CA Cancer J Clin 61: 69-90, 2011.

7. Iborra FJ, Kimura $\mathrm{H}$ and Cook PR: The functional organization of mitochondrial genomes in human cells. BMC Biol 2: 9, 2004.

8. Wallace DC: Mitochondria and cancer. Nat Rev Cancer 12: 685-698, 2012.

9. Shadel GS: Expression and maintenance of mitochondrial DNA New insights into human disease pathology. Am J Pathol 172: 1445-1456, 2008.

10. Chinnery PF and Hudson G: Mitochondrial genetics. Br Med Bull 106: 135-159, 2013.

11. Lightowlers RN, Chinnery PF, Turnbull DM and Howell N: Mammalian mitochondrial genetics: Heredity, heteroplasty and disease. Trends Genet 13: 450-455, 1997.

12. Copeland WC, Wachsman JT, Johnson FM and Penta JS: Mitochondrial DNA alterations in cancer. Cancer Invest 20: 557-569, 2002.

13. Zhang G, Qu Y, Dang S, Yang Q, Shi B and Hou P: Variable copy number of mitochondrial DNA (mtDNA) predicts worse prognosis in advanced gastric cancer patients. Diagn Pathol 8: 173,2013

14. Dang S, Qu Y, Wei J, Shao Y, Yang Q, Ji M, Shi B and Hou P: Low copy number of mitochondrial DNA (mtDNA) predicts worse prognosis in early-stage laryngeal cancer patients. Diagn Pathol 9: 28, 2014.

15. Zhang Y, Qu Y, Gao K, Yang Q, Shi B, Hou P and Ji M: High copy number of mitochondrial DNA (mtDNA) predicts good prognosis in glioma patients. Am J Cancer Res 5: 1207-1216, 2015.

16. Reznik E, Miller ML, Senbabaoğlu Y, Riaz N, Sarungbam J, Tickoo SK, Al-Ahmadie HA, Lee W, Seshan VE, Hakimi AA and Sander C: Mitochondrial DNA copy number variation across human cancers. Elife 5: pii:e10769, 2016.

17. Lan Q, Lim U, Liu CS, Weinstein SJ, Chanock S, Bonner MR, Virtamo J, Albanes D and Rothman N: A prospective study of mitochondrial DNA copy number and risk of non-Hodgkin lymphoma. Blood 112: 4247-4249, 2008.

18. Xing J, Chen M, Wood CG, Lin J, Spitz MR, Ma J, Amos CI, Shields PG, Benowitz NL, Gu J, et al: Mitochondrial DNA content: Its genetic heritability and association with renal cell carcinoma. J Natl Cancer Inst 100: 1104-1112, 2008.

19. Bonner MR, Shen M, Liu CS, Divita M, He X and Lan Q: Mitochondrial DNA content and lung cancer risk in Xuan Wei, China. Lung Cancer 63: 331-334, 2009.

20. Shen J, Platek M, Mahasneh A, Ambrosone CB and Zhao H: Mitochondrial copy number and risk of breast cancer: A pilot study. Mitochondrion 10: 62-68, 2010.

21. Liao LM, Baccarelli A, Shu XO, Gao YT, Ji BT, Yang G, Li HL, Hoxha M, Dioni L, Rothman N, et al: Mitochondrial DNA copy number and risk of gastric cancer: A report from the Shanghai Women's Health Study. Cancer Epidemiol Biomarkers Prev 20: 1944-1949, 2011

22. Lynch SM, Weinstein SJ, Virtamo J, Lan Q, Liu CS, Cheng WL, Rothman N, Albanes D and Stolzenberg-Solomon RZ: Mitochondrial DNA copy number and pancreatic cancer in the alpha-tocopherol beta-carotene cancer prevention study. Cancer Prev Res (Phila) 4: 1912-1919, 2011.

23. Shen L, Wei J, Chen T, He J, Qu J, He X, Jiang L, Qu Y, Fang H, Chen $\mathrm{G}$, et al: Evaluating mitochondrial DNA in patients with breast cancer and benign breast disease. J Cancer Res Clin Oncol 4: 669-675, 2011.

24. Hosgood HD III, Liu CS, Rothman N, Weinstein SJ, Bonner MR, Shen M, Lim U, Virtamo J, Cheng WL, Albanes D and Lan Q: Mitochondrial DNA copy number and lung cancer risk in a prospective cohort study. Carcinogenesis 31: 847-849, 2010.

25. Hamiltion SR and Aaltonen LA: Pathology and genetics of tumours of the digestive system. Lyon, IARCPress, 11-36, 2000.

26. Shi J, Yao D, Liu W, Wang N, Lv H, Zhang G, Ji M, Xu L, He N, Shi B and Hou P: Highly frequent PIK3CA amplification is associated with poor prognosis in gastric cancer. BMC Cancer 12: $50,2012$.

27. Stahl M, Mariette C, Haustermans K, Cervantes A and Arnold D; ESMO Guidelines Working Group: Oesophageal cancer: ESMO clinical practice guidelines for diagnosis, treatment and follow-up. Ann Oncol 24 (Suppl 6): vi51-vi56, 2013.

28. McBride HM, Neuspiel M and Wasiak S: Mitochondria: More than just a powerhouse. Curr Biol 16: R551-R560, 2006.

29. Gasparre G, Romeo G, Rugolo M and Porcelli AM: Learning from oncocytic tumors: Why choose inefficient mitochondria? Biochim Biophys Acta 1807: 633-642, 2011. 
30. Iommarini L, Calvaruso MA, Kurelac I, Gasparre G and Porcelli AM: Complex impairment in mitochondrial diseases and cancer: Parallel roads leading to different outcomes. Int J Biochem Cell Biol 45: 47-63, 2013.

31. Campbell CL and Thorsness PE: Escape of mitochondrial DNA to the nucleus in ymel yeast is mediated by vacuolar-dependent turnover of abnormal mitochondrial compartments. J Cell Sci 111: 2455-2564, 1998

32. Thorsness PE and Weber ER: Escape and migration of nucleic acids between chloroplasts, mitochondria, and the nucleus. Int Rev Cytol 165: 207-234, 1996.

33. Liang BC and Hays L: Mitochondrial DNA copy number changes in human gliomas. Cancer Lett 2: 167-173, 1996.

34. Netter P and Robineau S: The differential over amplification of short sequences in the mitochondrial DNA of rho-petites in Saccharomyces cerevisiae stimulates recombination. Gene 83: 25-38, 1989.

35. Eppert K, Scherer SW, Ozcelik H, Pirone R, Hoodless P, Kim H, Tsui LC, Bapat B, Gallinger S, Andrulis IL, et al: MADR2 maps to $18 \mathrm{q} 21$ and encodes a TGFbeta-regulated MAD-related protein that is functionally mutated in colorectal carcinoma. Cell 86 : 543-552, 1996.

36. Borensztajn K, Chafa O, Alhenc-Gelas M, Salha S, Reghis A, Fischer AM and Tapon-Bretaudière J: Characterization of two novel splice site mutations in human factor VII gene causing severe plasma factor VII deficiency and bleeding diathesis. Br J Haematol 117: 168-171, 2002.
37. TurnerC, Killoran C, Thomas NS, Rosenberg M, Chuzhanova NA, Johnston J, Kemel Y, Cooper DN and Biesecker LG: Human genetic disease caused by de novo mitochondrial-nuclear DNA transfer. Hum Genet 112: 303-309, 2003.

38. Zhang G, Qu Y, Dang S, Yang Q, Shi B and Hou P: Variable copy number of mitochondrial DNA (mtDNA) predicts worse prognosis in advanced gastric cancer patients. Diagn Pathol 8: 173, 2013.

39. Dang S, Qu Y, Wei J, Shao Y, Yang Q, Ji M, Shi B and Hou P: Low copy number of mitochondrial DNA (mtDNA) predicts worse prognosis in early-stage laryngeal cancer patients. Diagn Pathol 9: 28, 2014.

40. Liu M, Hu P, Zhang G, Zeng Y, Yang H, Fan J, Jin L, Liu H, Deng Y, Li S, et al: Copy number variation analysis by ligation-dependent PCR based on magnetic nanoparticles and chemiluminescence. Theranostics 4: 71-85, 2015.

41. Yang H, Liu M, Zhou B, Deng Y, He N, Jiang H, Guo Y, Lan G, Jiang Q, Yang $X$ and Li Z: Chemiluminescent detection for estimating relative copy numbers of porcine endogenous retrovirus proviruses from Chinese Minipigs based on magnetic nanoparticles. J Nanosci Nanotechnol 16: 6505-6510, 2016. Attribution-NonCommercial-NoDerivatives 4.0 International (CC BY-NC-ND 4.0) License. 